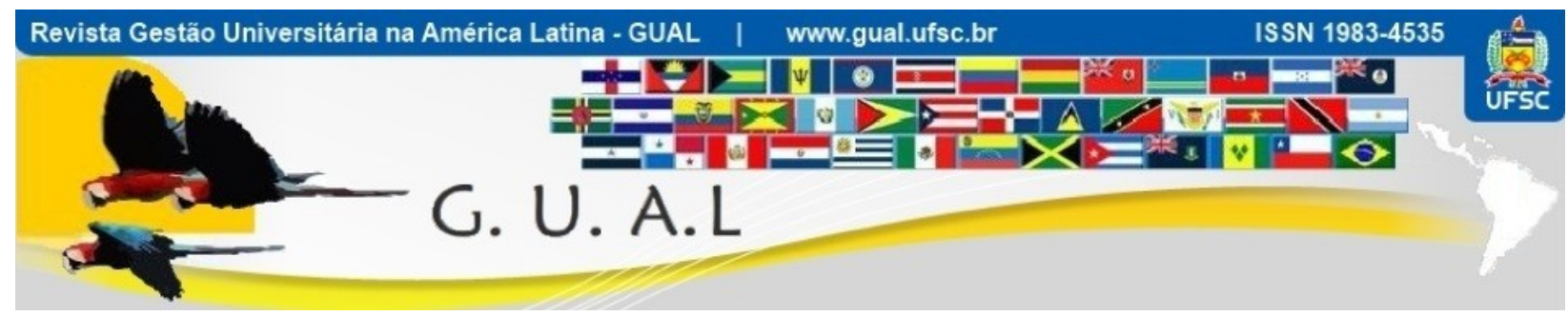

DOI: http://dx.doi.org/10.5007/1983-4535.2015v8n2p278

\title{
A AVALIAÇÃO DE PROGRAMAS DA CAPES, OS CONCEITOS E O USO DE INDICADORES BIBLIOGRÁFICOS
}

\author{
CAPES' PROGRAM EVALUATION, GRADES AND THE USE OF \\ BIBLIOGRAPHIC PRODUCTION INDICATORS
}

Sandro Vieira Soares, Doutorando

Universidade de São Paulo - USP

sandrovs@usp.br

Silvia Pereira de Castro Casa Nova, Doutora Universidade de São Paulo - USP silvianova@usp.br

Agradecimentos: Os autores agradecem Amélia Silveira, Cibele Barsalini Martins, Flavio Hourneaux Junior e Tobias Coutinho Parente pelos comentários e sugestões feitos durante a apresentação do artigo no XVII SemeAd.

Recebido em 24/outubro/2014

Aprovado em 17/março/2015

Sistema de Avaliação: Double Blind Review

Esta obra está sob uma Licença Creative Commons Atribuição-Uso. 


\title{
A AVALIAÇÃO DE PROGRAMAS DA CAPES, OS CONCEITOS E O USO DE INDICADORES BIBLIOGRÁFICOS \\ DOI: http://dx.doi.org/10.5007/1983-4535.2015v8n2p278
}

\section{RESUMO}

É ponto pacífico a necessidade de avaliar o desempenho dos programas quanto a sua capacidade de criar conhecimento que seja ratificado pela comunidade científica. Esse processo acontece por meio da publicação definitiva da produção em periódicos. Este artigo tem por objetivo investigar o impacto do uso dos indicadores de produção bibliográfica veiculada em periódicos no agrupamento dos programas de pós-graduação da área de Administração, Ciências Contábeis e Turismo no triênio 2010-2012. Os resultados mostraram que o agrupamento por análise de clusters resultou na criação de grupos altamente distintos quanto à sua composição qualitativa e quantitativa: os grupos 1, 2, 3, 4 e 5 foram formados por $8,3,3,13$ e 50 programas, respectivamente. Os programas com doutorado foram alocados principalmente aos grupos 1, 3 e 4 e os que possuem somente mestrado foram alocados todos no grupo 5. A análise de correspondência identificou associação entre cada grupo e os conceitos Capes. Foi possível identificar que o conceito do programa está apenas parcialmente relacionado à composição dos grupos com base em indicadores bibliográficos. Portanto, outros quesitos da avaliação, como formação de discípulos e composição do corpo docente, parecem ter um peso relativo relevante.

Palavras-chave: Pós-Graduação. Avaliação de programas. Conceito Capes. Indicadores bibliográficos. Produção Científica.

\begin{abstract}
The need to evaluate graduate programs' performance due to their capacity to add knowledge ratified by the scientific community is well accepted and understood. This paper aims to investigate the impact of the use of bibliographic production's indicators in the grouping of graduate programs in the Business, Accounting and Tourism knowledge area in the period of 2010-2012. The results revealed that grouping programs based on clusters analysis resulted in highly distinct clusters in terms of both their qualitative and quantitative composition. Groups 1, 2, 3, 4 and 5 were formed by 8, 3, 3, 13 and 50 programs, respectively. Programs with Master and $\mathrm{PhD}$ degrees were mainly allocated to groups 1,3 and 4 and programs with only Master degree have been allocated in the cluster 5. The correspondence analysis identified that those groups are associated to their Capes' grades. It was possible to identify that the program grade is only partially related to the composition of the clusters created using bibliographic indicators. Thus, other assessment criteria as master and $\mathrm{PhD}$ formation and faculty, seem to have a relevant relative weight.
\end{abstract}

Keywords: Graduate Programs. Program evaluation. Capes'grades. Bibliometric indicators. Scientific Production. 


\section{A AVALIAÇÃO DE PROGRAMAS DA CAPES, OS CONCEITOS E O USO DE INDICADORES \\ BIBLIOGRÁFICOS \\ DOI: http://dx.doi.org/10.5007/1983-4535.2014v7n2p1}

\section{INTRODUÇÃO}

A Coordenação de Aperfeiçoamento de Pessoal de Nível Superior (CAPES) é o órgão do Ministério da Educação (MEC) responsável pela avaliação dos programas de pósgraduação stricto sensu - Doutorado e Mestrado Acadêmicos e Mestrado Profissional - no Brasil. Atualmente a avaliação sobre o desempenho dos Programas é realizada trienalmente. Conceitos são atribuídos aos programas de acordo com o seu desempenho. As notas utilizadas são números de 1 a 7 e são definidos conforme o cumprimento de pré-requisitos estabelecidos para os cinco itens avaliados de cada programa (quesitos), que são: i. a Proposta do Programa, ii. Corpo Docente; iii. Corpo Discente, Teses e Dissertações; iv. Produção Intelectual e v. Inserção Social. Estes quesitos têm pesos arbitrariamente atribuídos a priori de $0 \%, 20 \%$, $35 \%, 35 \%$ e $10 \%$, respectivamente, que podem ser modificados entre trienais. O quesito com peso 0\% (Proposta do Programa) passa por uma avaliação qualitativa, tendo em vista sua coerência, histórico e planos de futuro.

Segundo o Relatório de Avaliação 2010-2012 da Trienal 2013 para um programa ser recomendado ou manter sua recomendação pela Capes, ele precisa ter seu desempenho avaliado com nota acima de 3 . Portanto, a nota 3 corresponde ao padrão mínimo de qualidade para a recomendação do programa ao Conselho Nacional de Educação (CNE) e consequente permanência no Sistema Nacional de Pós-Graduação (SNPG). A nota 4 é dada aos cursos que tenham alcançado, no mínimo, "Bom" em pelo menos três quesitos, incluindo, necessariamente, Corpo Discente e Trabalhos de Conclusão e Produção Intelectual (quesitos 3 e 4 ). A nota 5 é dada aos cursos que obtiverem "Muito Bom" em pelo menos quatro dos cinco quesitos existentes, entre os quais terão que figurar necessariamente os quesitos 3 e 4 . E, finalmente, as notas 6 e 7 são reservadas exclusivamente para os programas com doutorado que obtiveram nota 5 e conceito "Muito Bom" em todos os quesitos (Proposta do Programa; Corpo Docente, Teses e Dissertações; Produção Intelectual e Inserção Social) da ficha de avaliação e que atendam, necessariamente, a três condições:

Nota 6: predomínio do conceito "Muito Bom" nos itens de todos os quesitos da ficha de avaliação, mesmo com eventual conceito "Bom" em alguns itens; nível de desempenho (formação de doutores e produção intelectual) diferenciado em relação aos demais programas da área; e desempenho equivalente ao dos centros internacionais de excelência na área (internacionalização e liderança).

Nota 7: conceito "Muito Bom" em todos os itens de todos os quesitos da ficha de avaliação; nível de desempenho (formação de doutores e produção intelectual) altamente diferenciado em relação aos demais programas da área; e desempenho 


\section{A AVALIAÇÃO DE PROGRAMAS DA CAPES, OS CONCEITOS E O USO DE INDICADORES \\ BIBLIOGRÁFICOS \\ DOI: http://dx.doi.org/10.5007/1983-4535.2014v7n2p1}

equivalente ao dos centros internacionais de excelência na área (internacionalização e liderança). (CAPES, 2013b).

O quesito iv. Produção Intelectual, que é alvo de análise neste artigo, que contribui com $35 \%$ do desempenho total e é composto por três itens: 4.1 Publicações qualificadas do Programa por Docente Permanente; 4.2 Distribuição de publicações qualificadas em relação ao corpo docente permanente do Programa; e 4.3. Produção técnica, patentes e outras produções consideradas relevantes; com pesos arbitrados de 50\%, 35\% e 15\%, respectivamente. Além de ter grande peso na avaliação do programa, apenas comparável com o do quesito iii. Corpo Discente, Teses e Dissertações, a Produção Intelectual é um indicador de resultado que internacionalmente tem sido considerado no ranqueamento de programas e de pesquisadores. Apesar de uma grita em torno das distorções que indicadores de publicação e de citações possam acarretar, ainda não se encontrou outro substituto. Portanto, cabe o questionamento de como ficariam agrupados os programas se apenas fosse considerado esse produto último da atividade intelectual: a publicação científica.

A questão que motiva esta pesquisa é: como se agrupam os programas de pósgraduação stricto sensu acadêmicos da área de Administração, Ciências Contábeis e Turismo segundo a produção bibliográfica veiculada em periódicos? A resposta a essa questão tem dois objetivos. O primeiro deles é identificar como se agrupam os programas isolando o fator produção científica dos demais utilizados na avaliação da Capes. O segundo objetivo é identificar como se associa o desempenho dos programas à sua produção científica veiculada em periódicos com o Conceito Capes.

A justificativa para esta pesquisa é a necessidade de avaliar o desempenho dos programas quanto a sua capacidade de criar conhecimento novo que seja ratificado pela comunidade científica. Também se justifica esta pesquisa por constituir uma tentativa de contribuição na discussão acerca do tão debatido sistema de avaliação da Capes.

\section{REVISÃO BIBLIOGRÁFICA}

Diversos são os estudos que têm analisado a capacidade de construção do conhecimento em programas de pós-graduação no Brasil. Alguns deles constituem a criação de sistemas paralelos de avaliação de programas independentes da Capes ou constituem críticas que visam o aprimoramento do sistema de avaliação da Capes como em Beuren e Souza (2008), Igarashi et al. (2008) e Lins Almeida e Bartholo Jr. (2011). 


\section{A AVALIAÇÃO DE PROGRAMAS DA CAPES, OS CONCEITOS E O USO DE INDICADORES \\ BIBLIOGRÁFICOS \\ DOI: http://dx.doi.org/10.5007/1983-4535.2014v7n2p1}

Em seu sítio eletrônico, a Capes expõe que "a Avaliação do Sistema Nacional de PósGraduação, na forma como foi estabelecida a partir de 1998, é orientada pela Diretoria de Avaliação/Capes e realizada com a participação da comunidade acadêmico-científica por meio de consultores ad hoc". Enfatiza ainda que "a avaliação é atividade essencial para assegurar e manter a qualidade dos cursos de Mestrado e Doutorado no país". (CAPES, 2014b). A instituição estabelece como objetivos da Avaliação: (1) "a certificação da qualidade da pós-graduação brasileira, referência para a distribuição de bolsas e recursos para o fomento à pesquisa"; (2) a "identificação de assimetrias regionais e de áreas estratégicas do conhecimento no SNPG para orientar ações de indução na criação e expansão de programas de pós-graduação no território nacional. (CAPES, 2014b).

Por fim, os documentos de área, as fichas de avaliação e os relatórios de avaliação são indicados pela instituição como sendo o trinômio que constitui os referenciais dos processos de avaliação, como detalhado no website da Capes:

\footnotetext{
Os documentos de área são referência para os processos avaliativos, tanto na elaboração e submissão de propostas de cursos novos quanto na avaliação trienal dos cursos em funcionamento. Neles estão descritos o estado atual, as características e as perspectivas, assim como os quesitos considerados prioritários na avaliação dos programas de pós-graduação pertencentes a cada uma das 48 áreas de avaliação.

Em conjunto com as Fichas de Avaliação e os Relatórios de Avaliação, os Documentos de Área constituem o trinômio que expressa os processos e os resultados da Avaliação Trienal. (CAPES, 2014b)
}

No que diz respeito a avaliação da produção científica dos programas de pósgraduação, o sistema de avaliação de programas da Capes, já exaustivamente questionado (SPAGNOLO e SOUZA, 2004; MOREIRA, HORTALE e HARTZ, 2004), desenvolveu um igualmente criticado sistema de avaliação da qualidade de periódicos (BEUREN e SOUZA, 2008; ROCHA-E-SILVA, 2009; SOARES, CASA NOVA, CASTRO JR., 2014), para fins de avaliação da produção bibliográfica dos programas, denominado Qualis-Periódicos. O Qualis é descrito pela Capes como sendo "o conjunto de procedimentos utilizados pela Capes para estratificação da qualidade da produção intelectual dos programas de pós-graduação". É afirmado ainda que "tal proceso foi concebido para atender necessidade específicas do sistema de avaliação e é baseado nas informações fornecidas por meio do aplicativo Coleta de Dados", conhecido como Coleta-Capes e substituído em 2014 pela Plataforma Sucupira. Com base nesse processo, é disponibilizada "uma lista com a classificação dos veículos utilizados pelos programas de pós-graduação para a divulgação de sua produção”. As informações do website da Capes ainda esclarecem que "a estratificação da qualidade dessa produção é 


\section{A AVALIAÇÃO DE PROGRAMAS DA CAPES, OS CONCEITOS E O USO DE INDICADORES \\ BIBLIOGRÁFICOS \\ DOI: http://dx.doi.org/10.5007/1983-4535.2014v7n2p1}

realizada de forma indireta", pela aferição "da qualidade dos artigos e outros tipos de produção, a partir da análise da qualidadade dos veículos de divulgação, ou seja, periódicos científicos". Finalmente, algumas características da classificação são detalhadas:

A classificação de periódicos é realizada pelas áreas de avaliação e passa por processo anual de atualização. Esses veículos são enquadrados em estratos indicativos da qualidade - A1, o mais elevado; A2; B1; B2; B3; B4; B5; C - com peso zero. (CAPES, 2014c)

Diversas pesquisas analisaram criticamente e propuseram melhores ao sistema de avaliação da qualidade de periódicos. A pesquisa de Beuren e Souza (2008) é um exemplo de pesquisa que constitui uma tentativa de aprimoramento do sistema de avaliação da Capes, mais especificamente do Qualis. Nesta pesquisa, os autores identificaram 50 periódicos internacionais de contabilidade disponíveis no Portal de Periódicos da Capes, analisaram características, como a indexação destes periódicos em bases como Scopus e ISI, e existência ou não do fator de impacto JCR. Com isso os autores propõem uma classificação de periódicos em três estratos: "A" para periódicos classificados no JCR do ISI; "B" para periódicos que obtivessem fatores de impacto calculados conforme o estudo, tomando como referência a base SCOPUS, com fator de impacto maior ou igual a 0,5 ; e "C" para periódicos que obtivessem fatores de impacto calculados conforme o estudo, tomando como referência a base SCOPUS, com fator de impacto menor que 0,5. No triênio 2004-2006 a estratificação de periódicos se dividia em Internacional, Nacional e Regional e nos estratos A, B e C. Evidentemente essa foi uma proposta de alteração a esse sistema, que é anterior à adoção da classificação nos estratos A1, A2, B1, B2, B3, B4, B5 e C.

Outras pesquisas analisaram os padrões de publicação de autores na área em eventos e periódicos, vinculando-as a características dos autores e de instituições. Leite Filho (2008) analisou os padrões de produtividade de autores em periódicos e congressos na área de contabilidade no Brasil. O autor analisou as publicações veiculadas em duas revistas e dois eventos de contabilidade entre os períodos de 1989 e 2004, totalizando 902 artigos. Seus principais achados foram que havia uma predominância de autores do gênero masculino, indícios de forte endogenia de instituições nos eventos e periódicos dos quais eram organizadores e editoras, e principalmente, uma forte concentração de autorias vinculadas a poucas instituições. A USP aparece como a instituição mais recorrente na publicação em três dos quatro meios analisados. Esses achados apontam para uma produção ainda incipiente e concentrada na área, dependente ainda de poucas intituições. 


\section{A AVALIAÇÃO DE PROGRAMAS DA CAPES, OS CONCEITOS E O USO DE INDICADORES \\ BIBLIOGRÁFICOS \\ DOI: http://dx.doi.org/10.5007/1983-4535.2014v7n2p1}

Souza et al. (2008) fizeram uma análise semelhante a de Leite Filho (2008), mas com quatro eventos e em um período distinto. As autoras analisaram 657 artigos apresentados nesses eventos entre 2006 e 2007, e identificaram poucas instituições, USP, UnB, Fucape e UFSC, concentraram grande parte das produções feitas em colaboração com outras instituições, o que apoia a manutenção do diagnóstico feito por Leite Filho (2008) para período anterior. As autoras identificaram a USP como tendo o maior número de artigos publicados. Interessante notar a ênfase na análise de eventos em que a produção ainda está em estágio intermediário.

Leite Filho (2010) também faz uma análise de produções bibliográficas mas, ao invés de analisar periódicos ou eventos, opta por analisar o currículo de docentes de programas de pós-graduação em Contabilidade. Os achados do autor corroboram com os das pesquisas de Leite Filho (2008) e Souza et al. (2008) ao apontar que a distribuição da publicação não se dá de forma uniforme, e afirma que um grupo de cinco dos 18 programas de pós-graduação em contabilidade foi responsável por $55 \%$ dos artigos publicados em periódicos e $54 \%$ dos artigos veiculados em eventos sendo, que $87 \%$ dos artigos publicados em periódicos o foram em revistas nacionais. Nesta pesquisa a USP aparece como a instituição mais produtiva, em números absolutos, em periódicos e congressos. Em consonância com os achados de estudos anteriores, a produção da área está concentrado em poucos programas, tanto no caso de periódicos quanto no caso de eventos, e altamente nacionalizada. Dessa forma, é necessário um esforço de internacionalização da pesquisa na área.

Vieira, Ensslin e Martins (2011) realizaram estudo acerca do perfil da produção científica dos docentes de departamentos de contabilidade em três universidades federais da região Sul do Brasil nos anos de 2008 e 2009. Os autores encontraram que alguns poucos docentes concentram grande parte das publicações em eventos e periódicos, embora não tenham mencionado nem a lei de Lotka nem a lei de elitismo de Price. Sobre os artigos publicados em periódicos, na UFPR, dois docentes são autores de 67\% dos artigos, na UFSC, quatro docentes são autores de 57\% e na UFRGS, três autores concentram 70\% dos artigos publicados. Vieira, Ensslin e Martins (2011) ainda constataram a inexistência de artigos publicados nos estratos mais altos do Qualis, que são os estratos A1 e A2. Ou seja, analisando-se departamentos, encontra-se o mesmo quadro obtido ao analisar-se a produção em eventos, periódicos ou com base no currículo Lattes dos docentes: a concentração da produção nas mãos de poucos, quer sejam pesquisadores, programas ou instituições. 


\section{A AVALIAÇÃO DE PROGRAMAS DA CAPES, OS CONCEITOS E O USO DE INDICADORES

Nascimento e Beuren (2011) fizeram uma análise das publicações sob a ótica das redes sociais de pesquisa no triênio 2007-2009. Os resultados da pesquisa mostram que a produção científica definitiva veiculada em periódicos pelos docentes permanentes dos programas analisados apresenta-se dispersa nas estratificações do Qualis CAPES, que a centralidade da rede social é ocupada pelo programa da USP, e que os programas de pósgraduação em ciências contábeis apresentam ligações fracas, esparsas e pouco densas. Podese concluir com a construção de parcerias de pesquisa é necessária para a área fortalecer-se.

Silva et al. (2012) também analisaram a produção dos docentes dos programas de pósgraduação em contabilidade sob a perspectiva de redes e concluíram que a USP e FURB foram os programas com maior pontuação-média por professor no Qualis (USP com 165 e 120 em 2007 e 2008 e FURB com 113 em 2009).

Outras três pesquisas analisam a pós-graduação com proposta diferentes: a avaliação qualitativa de como as estratégias e os recursos influenciaram o desempenho segundo Martins (2013) e a criação de rankings segundo Costa (2011) e Soares, Richartz e Murcia (2013).

$\mathrm{Na}$ pesquisa de Martins (2013), a autora optou por avaliar os programas de pósgraduação da área 27 da Capes, ou seja, os programas da área de Administração, Ciências Contábeis e Turismo, sem distinguir os programas acadêmicos dos profissionais, no período de 2001 e 2009, por meio de pesquisa documental e de campo. A pesquisa documental identificou que a maioria dos programas que apresentaram trabalhos concluídos sistematicamente conquistaram notas melhores ao final de cada avaliação, que alterações (para mais ou para menos) no quadro de docente permanente dos programas obtiveram aumento de nota no último triênio avaliado e que com as redes de colaborações houve o fortalecimento das discussões de estudos e, consequentemente, de produções mais consistentes e mais publicações em periódicos e eventos pontuados no Qualis. A autora constatou ainda que os programas que progrediram consecutivamente entre os anos de 2001 a 2009 e obtiveram as notas 6 e 7 no triênio de 2007-2009, tiveram como princípio em suas tomadas de decisões, que inclui a escolha da estratégia a ser adotada, os critérios do sistema de avaliação da Capes.

Na pesquisa de Costa (2011) o autor criou um ranking para descrever as características da produção intelectual dos docentes vinculados aos 18 programas de pós-graduação em ciências contábeis em periódicos no período de 2000 a 2009. O autor identificou 27 periódicos impressos e eletrônicos disponíveis online e os 1.175 artigos que os docentes dos 


\section{A AVALIAÇÃO DE PROGRAMAS DA CAPES, OS CONCEITOS E O USO DE INDICADORES \\ BIBLIOGRÁFICOS \\ DOI: http://dx.doi.org/10.5007/1983-4535.2014v7n2p1}

programas de pós-graduação em contabilidade publicaram nesses periódicos. Nesta pesquisa, o autor identificou que cinco de 18 programas (USP, FURB, FUCAPE, UFMG e USP RP) são responsáveis por mais de $50 \%$ de toda produção docente no período o que vai ao encontro da pesquisa de Leite Filho (2010). O PPGCC/USP se mostrou como o programa com o melhor desempenho no ranking de Costa (2011) que levou em consideração, além da publicação total e por estrato Qualis, o desempenho por estratégia de pesquisa e por área temática. Comparando-se com pesquisas semelhantes, realizadas para períodos distintos, pode-se afirmar que apesar de ainda concentrada em poucos programas, aumentou o número de programas que comandam essa produção, inclusive alcançando uma certa dispersão em termos de estados (São Paulo, Santa Catarina, Espírito Santo e Minas Gerais), porém sem ainda incluir estados da região Nordeste, Centro-Oeste e Norte do país.

Na pesquisa de Soares, Richartz e Murcia (2013) os autores criaram um ranking dos 18 programas em Contabilidade segundo as publicações dos docentes para o triênio de 20072009. Os autores se utilizaram de cinco critérios para constituir seu ranking: i. pontuação total do programa; ii. pontuação média por professor; iii. coeficiente de variação da pontuação; iv. percentual de professores produtivos e v. nível de inserção internacional. Nesta pesquisa o PPGCC/USP encabeça os indicadores i., ii., iii. e iv. e a FURB encabeça o indicador v.. Mas muitas críticas podem ser tecidas à essa forma de ranking, pois que os pesquisadores consideraram como produção do programa, o somatório das produções individuais dos docentes, de forma que artigos com mais de um docente como autor foram contados mais de uma vez no somatório. Ou seja, houve duplicidade de contagem. Por outro lado, nesse ranking, os autores incluem um divisor da produção pelo número de docentes, o que equilibra o fato de que os programas possuem números distintos de docentes permanentes, o que torna mais adequada a comparação entre programas de distintos portes.

De forma geral, o que se depreende da análise das pesquisas anteriores sobre o tema é a centralidade do PPGCC/USP, o que se explica por sua tradição e porte, e a ênfase dos pesquisadores em avaliar os programas com base nas publicações de seus docentes, em eventos e periódicos, o que prioriza um indicador de resultado e não considera indicadores de processo. Salienta-se ainda as estratégias adotadas pelos programas focadas nos critérios de avaliação da Capes, o que pode levar a uma visão imediatista e que tem a nota como ponto focal. Outro ponto que as pesquisas reproduzem é uma avaliação calcada em indicadores quantitativos, o que encontra eco em uma grita em torno da adoção de indicadores de 


\section{A AVALIAÇÃO DE PROGRAMAS DA CAPES, OS CONCEITOS E O USO DE INDICADORES \\ BIBLIOGRÁFICOS \\ DOI: http://dx.doi.org/10.5007/1983-4535.2014v7n2p1}

qualidade. Finalmente, na análise temporal que essas pesquisas permitem, depreende-se que a área tem crescido e outros programas aparecem ao lado da USP como referências. O crescimento da área possibilita a criação de parcerias de pesquisa entre pesquisadores de diferentes programas, o que traz uma possibilidade de fortalecimento.

\section{METODOLOGIA}

Os dados utilizados nesta pesquisa foram coletados no sítio eletrônico avaliacaotrienal2013.capes.gov.br/ referentes ao triênio 2010-2012. Os dados originais das planilhas de indicadores foram atualizados pela última vez em 22 de abril de 2014 e conferidos quanto a sua atualidade em 26 de julho de 2014. Estes dados continham os indicadores dos 121 programas de pós-graduação stricto sensu recomendados pela Capes e avaliados quanto ao seu desempenho no triênio 2010-2012. Mas, nesta pesquisa, foram utilizados os dados exclusivamente dos 78 programas da modalidade acadêmica, desconsiderando assim os dados referentes aos 43 programas da modalidade profissional (CAPES, 2013a, 2014a).

Essa escolha metodológica se deu porquê os mestrados profissionais nasceram com uma proposta didático-pedagógica distinta dos mestrados acadêmicos e não é apropriado fazer uma análise dos indicadores de produção bibliográfica de programas criados com propostas distintas quanto a esse indicador, principalmente pelo fato de que os programas profissionais limitam-se tão somente ao nível de mestrado enquanto os acadêmicos englobam o nível de doutorado também. O natureza do produto que se espera nos programas profissionais é distinta dos programas acadêmicos (artigos, teses, dissertações). Assim, entende-se que a comparação entre programas de modalidades distintas seria inconsistente.

Os dados utilizados incluem basicamente a quantidade de pesquisas veiculadas em periódicos. As planilhas de indicadores contêm o quantitativo de "artigos completos publicados em periódicos técnico-científicos" discriminando essas publicações nos estratos $\mathrm{A} 1, \mathrm{~A} 2, \mathrm{~B} 1, \mathrm{~B} 2, \mathrm{~B} 3, \mathrm{~B} 4, \mathrm{~B} 5, \mathrm{C}$ e $\mathrm{NC}$ (não consta no Qualis). No referido triênio, os programas veicularam um total de 10.802 artigos em periódicos. No entanto, os artigos veiculados em revistas $\mathrm{NC}$, que foram 61 , representando $0,005 \%$ do total, não foram utilizados para a organização dos clusters.

A estatística descritiva da produção bibliográfica dos programas, por estrato, é evidenciada na Tabela 1: 


\section{A AVALIAÇÃO DE PROGRAMAS DA CAPES, OS CONCEITOS E O USO DE INDICADORES \\ BIBLIOGRÁFICOS \\ DOI: http://dx.doi.org/10.5007/1983-4535.2014v7n2p1}

Tabela 1 Estatística descritiva dos dados utilizados para agrupamento dos programas, por estrato

\begin{tabular}{l|c|c|c|c|c|c|c|c}
\hline Estrato & $\mathbf{A 1}$ & $\mathbf{A 2}$ & $\mathbf{B 1}$ & $\mathbf{B 2}$ & $\mathbf{B 3}$ & $\mathbf{B} 4$ & $\mathbf{B 5}$ & $\mathbf{C}$ \\
\hline Média & 4,37 & 19,56 & 32,85 & 22,09 & 30,45 & 11,74 & 11,45 & 5,19 \\
\hline Erro padrão & 0,76 & 2,29 & 2,88 & 2,08 & 2,66 & 1,18 & 1,00 & 0,64 \\
\hline Mediana & 1 & 12 & 24,5 & 16 & 24 & 8,5 & 10 & 3 \\
\hline Moda & 0 & 5 & 28 & 12 & 10 & 3 & 9 & 2 \\
\hline Desvio padrão & 6,70 & 20,20 & 25,46 & 18,36 & 23,51 & 10,41 & 8,79 & 5,64 \\
\hline $\begin{array}{l}\text { Variância da } \\
\text { amostra }\end{array}$ & 44,89 & 408,02 & 648,11 & 337,15 & 552,77 & 108,45 & 77,26 & 31,85 \\
\hline $\begin{array}{l}\text { Coeficiente de } \\
\text { variação }\end{array}$ & 153.32 & 103.27 & 77.50 & 83.11 & 77.21 & 88.67 & 76.77 & 108.67 \\
\hline Curtose & 7,56 & 3,90 & 4,09 & 3,45 & 2,14 & 2,91 & 3,72 & 4,90 \\
\hline Assimetria & 2,55 & 1,90 & 1,84 & 1,80 & 1,40 & 1,58 & 1,56 & 2,16 \\
\hline Intervalo & 36 & 96 & 139 & 87 & 120 & 53 & 48 & 27 \\
\hline Mínimo & 0 & 0 & 3 & 1 & 1 & 1 & 0 & 0 \\
\hline Máximo & 36 & 96 & 142 & 88 & 121 & 54 & 48 & 27 \\
\hline Soma & 341 & 1526 & 2562 & 1723 & 2375 & 916 & 893 & 405 \\
\hline Contagem & 78 & 78 & 78 & 78 & 78 & 78 & 78 & 78 \\
\hline
\end{tabular}

Pode-se verificar que os programas conseguiram publicar muitos artigos em revistas de estrato B1 a B3 (62\%). A moda do estrato A1 é zero e do estrato A2 é 5. Isso pode ser explicado porque não há nenhuma revista brasileira de Administração, Contabilidade ou Turismo classificada como A1, mas há várias classificadas como A2. Os extremos também permitem duas constatações interessantes: cada um dos 78 programas publicou no mínimo 1 artigo entre os estratos B1 e B4 mas isso já não ocorreu nos estratos A1, A2 e B5. Houve programas ainda que conseguiram publicar 36 artigos em A1 e 96 em A2.

Deve-se observar que 21 dos 78 programas (cerca de 27\%) não publicaram artigos em revistas A1, um programa não publicou artigos em revistas $\mathrm{A} 2$ e um programa não publicou artigo em revistas A. Por outro lado, dois programas não publicaram artigos em revistas B5, e seis programas não publicaram em revistas C.

Encabeçando as publicações em cada estrato tem-se a FGV/RJ_ADM_E com 36 artigos A1, a FGV/SP_ADM_E com 96 artigos de estrato A2, USP_ADM_E com 142 artigos no B1, com 121 artigos no B3, com 54 artigos no B4 e com 48 artigos no B5, FURB_CONT com 88 artigos no B2, e UFRJ_ADM_E com 27 artigos no estrato C.

Multiplicando-se cada artigo publicado em cada estrato, por cada programa, pelo peso que cada estrato possui (exemplo: A1 = 100) têm-se que os dez programas com maior pontuação são: USP_ADM_E (26.420), UFRGS_ADM_E (18.480), FGV/RJ_ADM_E (15.610), FURB_CONT (15.590), FGV/SP_ADM_E (15.210), Uninove_ADM_E (14.810), 


\section{A AVALIAÇÃO DE PROGRAMAS DA CAPES, OS CONCEITOS E O USO DE INDICADORES \\ BIBLIOGRÁFICOS \\ DOI: http://dx.doi.org/10.5007/1983-4535.2014v7n2p1}

UFMG_ADM_E (14.760), USP_CONT (14.310), UFSM_ADM_E (12.680) e UFSC_CONT (12.120).

Deve se levar em consideração, ao se analisar a Tabela 1, que 33 programas acadêmicos ainda não possuem doutorado. Apenas 27 destes programas possuíam doutorados em funcionamento até 2009, 9 tiveram doutorado com início durante o triênio, e 9 programas em 2013.

Foi ainda calculada a correlação entre tamanho de programa e a produção bibliográfica, conforme Tabela 2, apresentada a seguir.

Tabela 2 Correlação entre Tamanho do programa e Produção bibliográfica

\begin{tabular}{c|c|c|c|c}
\hline & Docentes & Teses & Dissertações & Artigos \\
\hline Docentes & 1 & & & \\
\hline Teses & 0,83 & 1 & & \\
\hline Dissertações & 0,42 & 0,36 & 1 & \\
\hline Artigos & 0,78 & 0,72 & 0,34 & 1 \\
\hline
\end{tabular}

Com isso identificou-se que há correlações positivas entre o número de docentes, o número de teses e o número de dissertações defendidas e o número total de artigos publicados nos periódicos. A correlação mais forte se deu entre o tamanho do corpo docente e o total de artigos publicados $(r=0,78)$, um resultado esperado e que demonstra que a avaliação deveria considerar diferenças de porte.

A correlação entre idade do programa e produção bibliográfica $(r=0,48)$, no entanto, não foi tão forte quanto o tamanho do corpo docente, como pode ser observado na Tabela 3.

Tabela 3 Correlação entre Idade do programa e Produção bibliográfica

\begin{tabular}{c|c|c|c|c}
\hline & Idade & Teses & Dissertações & Artigos \\
\hline Idade & 1 & & & \\
\hline Teses & 0,61 & 1 & & \\
\hline Dissertações & 0,59 & 0,36 & 1 & \\
\hline Artigos & 0,48 & 0,72 & 0,34 & 1 \\
\hline
\end{tabular}

A explicação para isso pode ser o fato de que a idade de um programa é um dos fatores que colaboram para a capacidade de produção bibliográfica importante dada a possibilidade de implementar e consolidar grupos de pesquisa, mas que concorre com outras variáveis como número de docentes, discentes e financiamento.

Interessante notar ainda a correlação positiva e relevante $(\mathrm{r}=0,78)$ entre o número de artigos e o número de teses, que não se repete se considerado o número de dissertações $(\mathrm{r}=$ 0,34). Esse resultado pode ser uma indicação de que o doutorado é na verdade o indutor do 


\section{A AVALIAÇÃO DE PROGRAMAS DA CAPES, OS CONCEITOS E O USO DE INDICADORES \\ BIBLIOGRÁFICOS \\ DOI: http://dx.doi.org/10.5007/1983-4535.2014v7n2p1}

conhecimento que encontrará vazão e reconhecimento nos periódicos na forma de produção definitiva.

A técnica utilizada para agrupamento dos programas foi a análise de conglomerados também conhecida como clusters analysis. Para Fávero et al. (2009) esta é uma técnica estatística de interdependência que permite agrupar casos ou variáveis em grupos homogêneos em função do grau de similaridades entre os indivíduos, a partir de variáveis predeterminadas, e que busca maximizar a homogeneidade dos objetos dentro dos grupos e maximizar a heterogeneidade entre os demais grupos.

A padronização das variáveis utilizada nesta análise foi a $\mathrm{Z}$ scores calculada pela equação:

$$
Z=\frac{(x-\text { média })}{\text { desvio }- \text { padrão }}
$$

$$
d_{i j}=\sqrt{\sum_{k=1}^{p}\left(x_{i k}-x_{j k}\right)^{2}}
$$

Tal método padroniza cada variável (x) de maneira a apresentar média zero e desvio-padrão 1 e é a mais utilizada neste tipo de análise (FÁVERO et al., 2009). A medida de distância utilizada foi a distância euclidiana, onde a distância entre duas observações (i e j) corresponde à raiz quadrada da soma dos quadrados das diferenças entre i e j para todas as $\mathrm{p}$ variáveis.

O método escolhido foi o não-hierárquico k-means que objetiva minimizar a variância interna aos grupos e maximizar a variância entre grupos. Tal método requer a definição prévia do número de agrupamentos que o pesquisador deseja estabelecer. Nesta pesquisa identificouse que era necessário que os programas analisados se agrupassem em cinco clusters ou grupos. Isso ocorreu porque os programas avaliados pela Capes são classificados em sete categorias de acordo com o conceito (de 1 a 7) que recebem pela avaliação. Os conceitos 1 e 2 levam o programa ao descredenciamento e os programas que receberam esta nota nem são evidenciados na planilha dos indicadores, de forma que os programas que constam nesta planilha receberam conceitos de 3 a 7, representando cinco categorias. $\mathrm{O}$ algoritmo utilizado para a determinação da alocação das observações em cada conglomerado no método k-means é denominado nearest centroid sorting. (FÁVERO et al., 2009)

A existência de outliers foi examinada com base no diagrama de dispersão, conforme mostra a Figura 1: 


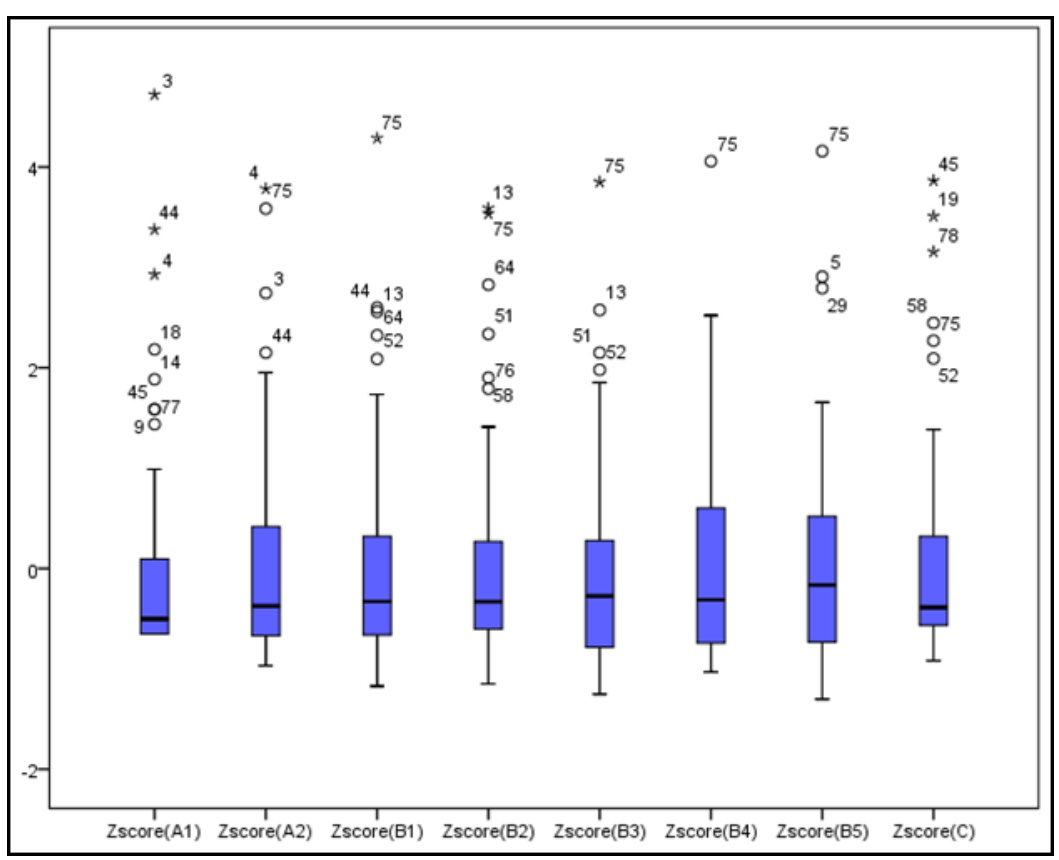

Figura 1 Diagramas de caixas para identificação de outliers

Os diagramas de caixas das distribuições evidenciam a existência possível e provável de um número tão grande de outliers que os pesquisadores optaram por não eliminar a maioria dos casos. Fávero et al. (2009) alertam que embora seja comum que indivíduos atípicos formem grupos isolados pode ser do interesse dos pesquisadores esta constatação de forma que a manutenção dos outliers na análise permite a observação do comportamento destes indivíduos.

O programa USP_ADM_E (75 na Figura 1) constitui um outlier e outras informações ajudam a ratificar esse diagnóstico como, por exemplo, o número de docentes permanentes (42 docentes, o maior número de docentes do país seguido pelo UFRGS_ADM_E com 34 docentes) e idade, já que o programa tem o doutorado mais antigo do país criado em 1975 (seguido da UFRJ_ADM_E e FGV/SP_ADM_E, ambos criados em 1976, e da USP_CONT de 1978). Isto também havia sido detectado quando foram realizadas análises de conglomerados hierárquicos de solução única de cinco clusters, usando distância quadrática euclidiana e ligação individual, completa, média e centróide. Este programa foi o único outlier removido da amostra.

Para atingir o segundo objetivo deste artigo, ainda foi utilizada a análise de correspondência que busca identificar a relação entre variáveis qualitativas, permitindo aos pesquisadores a visualização de associações. O mapa perceptual gerado pela referida técnica é mostrado no final da próxima seção. 


\section{A AVALIAÇÃO DE PROGRAMAS DA CAPES, OS CONCEITOS E O USO DE INDICADORES

\subsection{LIMITAÇÕES}

Primeiramente deve-se mencionar a limitação das duas técnicas utilizadas, já que se tratam de técnicas exploratórias altamente sensíveis àa inclusão ou à exclusão de observações.

Os dados utilizados foram os disponibilizados pelas Capes no sítio eletrônico avaliacaotrienal2013.capes.gov.br/ e assumiu-se que estes são dados verídicos e não comportam erros.

Também optou-se por analisar os dados dos programas de Administração, Ciências Contábeis e Turismo em conjunto, embora sejam programas com objetos de estudo e demandas distintos (SOARES et al., 2011; SOARES e PFITSCHER, 2012).

Ainda há três limitações de maior importância originadas pelas escolhas metodológicas feitas pelos autores. A primeira delas trata-se da eliminação dos programas da modalidade profissional da amostra. Dado que a análise de conglomerados é altamente sensível à inclusão ou à exclusão de indivíduos, os clusters se considerados os programas profissionais na base de dados certamente se organizariam de modo bastante distinto.

A segunda escolha metodológica dos autores que certamente acarretou alto impacto na análise de resultado foi a definição do número de categorias da análise não-hierárquica de clusters. O número de cinco categorias dos clusters foi definido exatamente porque também este é o número de categorias em que se dividem os programas recomendados pela Capes.

A terceira escolha metodológica de alto impacto para a pesquisa foi a manutenção dos outliers detectados pela análise dos diagramas de dispersão. A manutenção deles foi feita propositalmente por dois motivos: o primeiro é que eles constituíam um número significativo de casos e segundo porque a técnica de análise de clusters é altamente sensível à exclusão de observações de modo que os autores julgaram inadequado excluir estas observações para obtenção de uma análise mais homogênea. Diante disto, os autores optaram por excluir apenas o outlier identificado pelos diagramas de dispersão e que ocupava um cluster isoladamente, o programa de administração da USP (USP_ADM_E), que foi excluído.

Outra limitação desta pesquisa é que não se corrigiu o desempenho do programa pelo tamanho do seu corpo docente permanente, como na pesquisa de Soares, Richartz e Murcia (2013) de modo que o programa de administração da UFRGS (UFRGS_ADM_E), que possui 34 docentes permanentes, foi comparado com programas com nove docentes, por exemplo. No entanto, a utilização dessa correção é necessária quando se pretende avaliar desempenho de programas, o que não constituía a proposta deste artigo. 


\section{A AVALIAÇÃO DE PROGRAMAS DA CAPES, OS CONCEITOS E O USO DE INDICADORES \\ BIBLIOGRÁFICOS \\ DOI: http://dx.doi.org/10.5007/1983-4535.2014v7n2p1}

\section{ANÁLISE DOS RESULTADOS}

A Tabela 4 evidencia a composição dos cinco clusters gerados pela análise nãohierárquica k-Means realizada com as variáveis padronizadas pelo método $\mathrm{Z}$ scores, a distância euclidiana, e o algoritmo nearest centroid sorting. Os nomes dos programas foram codificados da seguinte forma: ies_área, ou seja, primeiramente aparece o nome da instituição de ensino superior e separado por um underline, a área do programa, para a qual adotou-se a seguinte codificação ADM_E = Administração de Empresas, ADM_P = Administração Pública, CONT $=$ Contabilidade e TURI $=$ Turismo.

Tabela 4 Composição dos Clusters

\begin{tabular}{|c|c|c|c|c|c|c|c|}
\hline N. & Programa & Cluster & Distância & N. & Programa & Cluster & Distância \\
\hline 13 & FURB_CONT & 1 & 2,471 & 20 & PUC/SP_CONT & 5 & 1,633 \\
\hline 36 & UFMG_ADM_E & 1 & 1,962 & 21 & UAM_TURI & 5 & 1,430 \\
\hline 50 & UFSC_ADM_E & 1 & 1,631 & 23 & UCS_TURI & 5 & 0,976 \\
\hline 51 & UFSC_CONT & 1 & 1,641 & 24 & UDESC_ADM_E & 5 & 1,105 \\
\hline 52 & UFSM_ADM_E & 1 & 2,623 & 25 & UECE_ADM_E & 5 & 1,423 \\
\hline 58 & UNB_CONT & 1 & 2,240 & 27 & UEM_ADM_E & 5 & 0,431 \\
\hline 64 & UNINOVE_ADM_E & 1 & 1,331 & 28 & UERJ_CONT & 5 & 0,949 \\
\hline 75 & USP_CONT & 1 & 1,759 & 30 & UFBA_CONT & 5 & 1,357 \\
\hline 19 & PUC/SP_ADM_E & 2 & 1,616 & 31 & UFC_ADM_E & 5 & 1,083 \\
\hline 45 & UFRJ_ADM_E & 2 & 1,764 & 32 & UFES_ADM_E & 5 & 0,950 \\
\hline 77 & USP/RP_CONT & 2 & 1,501 & 33 & UFES_CONT & 5 & 0,374 \\
\hline 3 & FGV/RJ_ADM_E & 3 & 1,535 & 34 & UFF ADM E & 5 & 1,364 \\
\hline 4 & FGV/SP_ADM_E & 3 & 1,954 & 37 & UFMG_CONT & 5 & 0,962 \\
\hline 44 & UFRGS_ADM_E & 3 & 2,127 & 38 & UFMS_ADM_E & 5 & 1,239 \\
\hline 5 & FGV/SP_ADM_P & 4 & 2,854 & 39 & UFPB/JP_ADM_E & 5 & 1,209 \\
\hline 18 & PUC/RS_ADM_E & 4 & 2,600 & 41 & UFPE_CONT & 5 & 0,989 \\
\hline 14 & PUC-RIO_ADM_E & 4 & 2,451 & 46 & UFRJ_CONT & 5 & 0,802 \\
\hline 22 & UCS_ADM_E & 4 & 2,232 & 47 & UFRN_ADM_E & 5 & 0,722 \\
\hline 26 & UEL_ADM_E & 4 & 2,276 & 48 & UFRN_TURI & 5 & 0,895 \\
\hline 29 & UFBA_ADM_E & 4 & 2,221 & 49 & UFRPE_ADM_E & 5 & 0,929 \\
\hline 35 & UFLA_ADM_E & 4 & 2,203 & 53 & UFU_ADM_E & 5 & 0,728 \\
\hline 40 & UFPE_ADM_E & 4 & 2,167 & 54 & UFV_ADM_E & 5 & 1,073 \\
\hline 42 & UFPR_ADM_E & 4 & 2,041 & 55 & UMESP_ADM_E & 5 & 0,911 \\
\hline 43 & UFPR_CONT & 4 & 2,138 & 56 & UNAMA_ADM_E & 5 & 1,090 \\
\hline 57 & UNB_ADM_E & 4 & 1,261 & 59 & UNIFACS_ADM_E & 5 & 1,113 \\
\hline 67 & UNISINOS_ADM_E & 4 & 1,446 & 60 & UniFECAP_CONT & 5 & 1,297 \\
\hline 76 & USP/RP_ADM_E & 4 & 2,149 & 61 & UNIFOR_ADM_E & 5 & 0,735 \\
\hline 1 & ESPM_ADM_E & 5 & 0,949 & 62 & UNIGRANRIO_ADM_E & 5 & 0,788 \\
\hline 2 & FEI_ADM_E & 5 & 1,100 & 63 & UNIMEP_ADM_E & 5 & 1,196 \\
\hline 6 & FJP_ADM_P & 5 & 2,010 & 65 & UNIP_ADM_E & 5 & 0,889 \\
\hline 7 & FNH_ADM_E & 5 & 0,842 & 66 & UNIR_ADM_E & 5 & 1,548 \\
\hline 8 & FUCAPE_ADM_E & 5 & 1,781 & 68 & UNISINOS_CONT & 5 & 1,642 \\
\hline 9 & FUCAPE_CONT & 5 & 2,369 & 69 & UNISUL_ADM_E & 5 & 0,926 \\
\hline 10 & FUFSE_ADM_E & 5 & 1,384 & 70 & UNIVALI_ADM_E & 5 & 1,700 \\
\hline 11 & FUMEC_ADM_E & 5 & 1,777 & 71 & UNIVALI_TURI & 5 & 0,835 \\
\hline 12 & FURB_ADM_E & 5 & 2,382 & 72 & UP_ADM_E & 5 & 1,627 \\
\hline 15 & PUC/MG_ADM_E & 5 & 0,943 & 73 & UPM_ADM_E & 5 & 1,361 \\
\hline 16 & PUC/PR_ADM_E & 5 & 1,478 & 74 & USCS_ADM_E & 5 & 1,578 \\
\hline
\end{tabular}




\begin{tabular}{c|l|c|c|c|l|c|c}
\hline N. & Programa & Cluster & Distância & N. & Programa & Cluster & Distância \\
\hline 17 & PUC/RS_ADM_E & 5 & 1,490 & & & & \\
\hline
\end{tabular}

A Tabela 5 agrupa os programas por cluster para melhor visualização. Cada grupo está indicado com o elenco dos programas que o compõe. Nesta Tabela mais duas informações foram evidenciadas. Programas com doutorado iniciado até 2009 estão em negrito. Pode-se notar que eles se concentram nos clusters 1, 3 e 4. Na Tabela 3 ainda são identificados os programas de Administração de Empresas em preto, Contabilidade em vermelho, de Administração Pública em verde e de Turismo em azul. Os programas de Contabilidade se concentram nos clusters 1 e 5, os de Administração Pública se dividem entre o 4 e 5 e os de Turismo se concentram totalmente no 5 .

Tabela 5 Composição dos clusters

\begin{tabular}{|c|c|c|c|c|}
\hline Clusters & \multicolumn{4}{|c|}{ Programas } \\
\hline 1 & $\begin{array}{l}\text { FURB_CONT } \\
\text { UFSC_CONT }\end{array}$ & $\begin{array}{l}\text { UNB_CONT } \\
\text { USP_CONT }\end{array}$ & $\begin{array}{l}\text { UFMG_ADM_E } \\
\text { UFSC_ADM_E }\end{array}$ & $\begin{array}{l}\text { UFSM_ADM_E } \\
\text { UNINOVE_ADM_E }\end{array}$ \\
\hline 2 & USP/RP_CONT & PUC/SP_ADM_E & UFRJ_ADM_E & \\
\hline 3 & FGV/RJ_ADM_E & FGV/SP_ADM_E & UFRGS_ADM_E & \\
\hline 4 & $\begin{array}{l}\text { UFPR_CONT } \\
\text { FGV/SP_ADM_P }\end{array}$ & $\begin{array}{l}\text { PUC/RS_ADM_E } \\
\text { PUC-RIO_ADM_E } \\
\text { UCS_ADM_E } \\
\text { UEL_ADM_E }\end{array}$ & $\begin{array}{l}\text { UFBA_ADM_E } \\
\text { UFLA_ADM_E } \\
\text { UFPE_ADM_E } \\
\text { UFPR_ADM_E }\end{array}$ & $\begin{array}{l}\text { UNB_ADM_E } \\
\text { UNISINOS_ADM_E } \\
\text { USP/RP_ADM_E }\end{array}$ \\
\hline 5 & $\begin{array}{l}\text { FUCAPE_CONT } \\
\text { PUC/SP_CONT } \\
\text { UERJ_CONT } \\
\text { UFBA_CONT } \\
\text { UFES_CONT } \\
\text { UFMG_CONT } \\
\text { UFPE_CONT } \\
\text { UFRJ_CONT } \\
\text { UniFECAP_CONT } \\
\text { UNISINOS_CONT } \\
\text { UAM_TURI } \\
\text { UCS_TURI } \\
\text { UFRN_TURI } \\
\text { UNIVALI_TURI }\end{array}$ & $\begin{array}{l}\text { FJP_ADM_P } \\
\text { ESPM_ADM_E } \\
\text { FEI_ADM_E } \\
\text { FNH_ADM_E } \\
\text { FUCAPE_ADM_E } \\
\text { FUFSE_ADM_E } \\
\text { FUMEC_ADM_E } \\
\text { FURB_ADM_E } \\
\text { PUC/MG_ADM_E } \\
\text { PUC/PR_ADM_E } \\
\text { PUC/RS_ADM_E } \\
\text { UDESC_ADM_E } \\
\text { UECE_ADM_E }\end{array}$ & $\begin{array}{l}\text { UEM_ADM_E } \\
\text { UFC_ADM_E } \\
\text { UFES_ADM_E } \\
\text { UFF_ADM_E } \\
\text { UFMS_ADM_E } \\
\text { UFPB/JP_ADM_E } \\
\text { UFRN_ADM_E } \\
\text { UFRPE_ADM_E } \\
\text { UFU_ADM_E } \\
\text { UFV_ADM_E } \\
\text { UMESP_ADM_E } \\
\text { UNAMA_ADM_E } \\
\text { UNIFACS_ADM_E } \\
\text { UNIFOR_ADM_E }\end{array}$ & $\begin{array}{l}\text { UNIGRANRIO_ADM_E } \\
\text { UNIMEP_ADM_E } \\
\text { UNIP_ADM_E } \\
\text { UNIR_ADM_E } \\
\text { UNISUL_ADM_E } \\
\text { UNIVALI_ADM_E } \\
\text { UP_ADM_E } \\
\text { UPM_ADM_E } \\
\text { USCS_ADM_E }\end{array}$ \\
\hline
\end{tabular}

Os clusters 1, 3 e 4 são formados majoritariamente por programas com doutorado iniciado até 2009 enquanto o cluster 5 é formado por programas que não possuíam doutorado antes do início do triênio. Já o cluster 2 possuía apenas um programa com doutorado iniciado até 2009 mas os outros dois programas abriram seus doutorados durante o triênio (2010 e 2012).

O segundo objetivo deste artigo é identificar como se associa o desempenho quanto a produção científica veiculada em periódicos com o desempenho global na forma do Conceito 


\section{A AVALIAÇÃO DE PROGRAMAS DA CAPES, OS CONCEITOS E O USO DE INDICADORES \\ BIBLIOGRÁFICOS \\ DOI: http://dx.doi.org/10.5007/1983-4535.2014v7n2p1}

Capes. Para tanto, fez-se uso da Análise de Correspondência tomando por variáveis qualitativas o conceito Capes de cada programa e associando este conceito aos grupos criados pela análise de conglomerados. O primeiro passo para se utilizar a análise de correspondência é testar, por meio do teste Qui-Quadrado se há associação entre as duas variáveis:

Tabela 6 Teste Qui-Quadrado de associação das variáveis Clusters e Conceito Capes

\begin{tabular}{l|c|c|c}
\hline & Valor & gl & Sig. Assint. (2-lados) \\
\hline Pearson Chi-Square & 55,311 & 16 & 0,000 \\
\hline Taxa de probabilidade & 34,717 & 16 & 0,004 \\
\hline Associação linear & 10,293 & 1 & 0,001 \\
\hline N. de Casos Válidos & 77 & & \\
a 20 células (80,0\%) têm esperado contagem inferior a 5. A contagem esperada é \\
mínimo, 03.
\end{tabular}

Estabelecido um nível de significância de 5\%, o teste Qui-quadrado indica um Asymp. Sig. $=0,000<0,05$, ou seja, rejeita-se a hipótese nula e aceita-se a hipótese de há associação estatisticamente significativa entre as duas variáveis.

A Tabela 7 mostra uma forte concentração dos programas entre conceitos 3 e 5 e uma forte concentração de programas nos clusters 1,4 e 5 .

Tabela 7 Tabela de contingência das variáveis cluster e conceito Capes

\begin{tabular}{c|c|c|c|c|c|c}
\hline & \multicolumn{7}{|c}{ Número do cluster } \\
\hline Conceito Capes & $\mathbf{1}$ & $\mathbf{2}$ & $\mathbf{3}$ & $\mathbf{4}$ & $\mathbf{5}$ & Total \\
\hline 3 & 0 & 0 & 0 & 1 & 14 & 15 \\
\hline 4 & 4 & 3 & 0 & 6 & 26 & 39 \\
\hline 5 & 2 & 0 & 1 & 6 & 10 & 19 \\
\hline 6 & 2 & 0 & 1 & 0 & 0 & 3 \\
\hline 7 & 0 & 0 & 1 & 0 & 0 & 1 \\
\hline Total & $\mathbf{8}$ & $\mathbf{3}$ & $\mathbf{3}$ & $\mathbf{1 3}$ & $\mathbf{5 0}$ & $\mathbf{7 7}$ \\
\hline
\end{tabular}

A Tabela 8 mostra que as dimensões 1 e 2 explicam respectivamente $69 \%$ e $17,6 \%$, da inércia total.

Tabela 8 Inércia e auto-valor

\begin{tabular}{c|c|c|c|c|c|c|c|c}
\hline \multirow{2}{*}{ Dimensão } & \multirow{2}{*}{$\begin{array}{c}\text { Valor } \\
\text { Singular }\end{array}$} & \multirow{2}{*}{ Inércia } & \multirow{2}{*}{$\begin{array}{c}\text { Chi } \\
\text { Square }\end{array}$} & \multirow{2}{*}{ Sig. } & \multicolumn{2}{|c}{ Proporção da Inércia } & \multicolumn{2}{|c}{ IC do VS } \\
\cline { 6 - 9 } & & & & Individual & Acumulada & $\begin{array}{c}\text { Desvio- } \\
\text { padrão }\end{array}$ & Correlação \\
\hline 1 & 0,705 & 0,497 & & & 0,692 & 0,692 & 0,139 & 0,031 \\
\hline 2 & 0,356 & 0,126 & & & 0,176 & 0,868 & 0,162 & \\
\hline 3 & 0,25 & 0,063 & & & 0,087 & 0,955 & & \\
\hline 4 & 0,179 & 0,032 & & & 0,045 & 1 & & \\
\hline Total & & 0,718 & 55,311 & 0 & 1 & 1 & & \\
\hline
\end{tabular}

a 16 graus de liberdade 
A Figura 2 representa o mapa perceptual das relações entre cluster e conceito Capes.

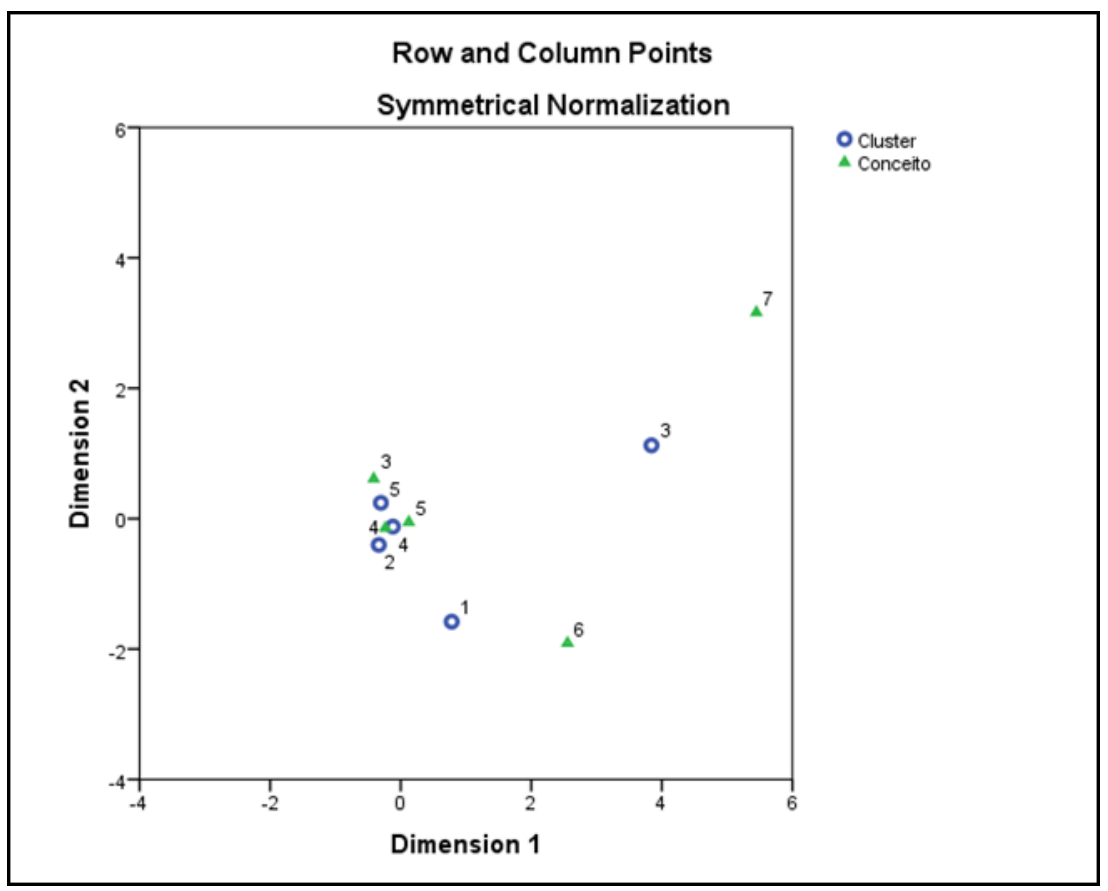

Figura 2 Mapa perceptual da relação cluster e conceito Capes

Fica claramente identificada a relação entre os conceitos 5, 6 e 7 e o pertencimento ao cluster 3. Vale lembrar que o cluster 3 é composto exclusivamente pelos programas de Administração de Empresas da FGV/SP, FGV/RJ e UFRGS. Os programas do cluster 1 estão alocados entre os conceitos 4,5 e 6 . Os programas do cluster 5 também ficam a aproximadamente a mesma distância dos conceitos 3, 4 e 5. O cluster 4 fica próximo dos conceitos 4 e 5 e o cluster 2 mais próximos do conceito 4 .

$\mathrm{Na}$ pesquisa de Martins (2013), a autora constatou ainda que os programas que progrediram consecutivamente entre os anos de 2001 a 2009 e obtiveram as notas 6 e 7 no triênio de 2007-2009, tiveram como princípio em suas tomadas de decisões os critérios do sistema de avaliação da Capes. Na presente pesquisa identifica-se que programas dos clusters 1 estão mais próximos do conceito 6 que os demais. Estes programas têm algumas características em comum, como por exemplo, a oferta de doutorado anterior ao início do triênio ( 7 dos 8 programas alocados no cluster 1 já ofertava doutorado).

Vale lembrar que o conceito 5, segundo o Relatório de Avaliação 2010-2012 Trienal 2013 (CAPES, 2013b, p. 7) “é a nota máxima admitida para programas que ofereçam apenas mestrado" o que torna coerente que os programas alocados no cluster 5, formado 


\section{A AVALIAÇÃO DE PROGRAMAS DA CAPES, OS CONCEITOS E O USO DE INDICADORES \\ BIBLIOGRÁFICOS \\ DOI: http://dx.doi.org/10.5007/1983-4535.2014v7n2p1}

predominantemente por programas que ofertam apenas mestrado, estejam ligados aos conceitos 3,4 e 5 .

$\mathrm{Na}$ pesquisa de Costa (2011) o autor destaca o desempenho dos programas de Contabilidade da USP, FURB, FUCAPE, UFMG e USP-RP como de melhores indicadores em seu ranking baseado no período compreendido entre 2000 e 2009. Com base exclusivamente na produção bibliográfica veiculada em periódicos em 2010-2012 este grupo se divide por clusters distintos: USP e FURB foram alocados ao cluster 1, USP/RP ao cluster 2, e FUCAPE e UFMG ao cluster 5.

Na pesquisa de Soares, Richartz e Murcia (2013) os autores destacaram o desempenho dos programas de Contabilidade da USP, FURB, FUCAPE, USP/RP e UFPE durante o período de 2007-2009. Em parte, seus achados convergem com a pesquisa de Costa (2011) e também avaliam como de melhor desempenho programas que se alocam em clusters distintos: USP e FURB no cluster 1, USP/RP no cluster 2 e FUCAPE e UFPE no cluster 5.

Evidentemente há que se considerar o cuidado de se deve ter com a análise de conglomerados dado que esta é uma técnica exploratória altamente sensível a inclusão de novos elementos e nas pesquisas de Costa (2011) e Soares, Richartz e Murcia (2013) os autores não consideraram os programas de Administração de Empresas e Pública e de Turismo aqui utilizados para o agrupamento o que provavelmente pode ter ajudado a separar esses programas em clusters distintos.

Deve-se notar que os clusters agruparam programas de conceitos bastante distintos: o cluster 1 reuniu programas com conceitos 4 a 6 , o cluster 3 reuniu programas com conceitos 5 a 7, os clusters 4 e 5 reuniram programas com conceitos 3 a 5 . O único cluster que apresentou grande homogeneidade interna em relação aos conceitos Capes foi o 2 que foi formados tão somente por três programas, todos com conceito 4 . Ou seja, a produção científica definitiva dos programas não foi capaz de os agrupar em clusters que sejam homogêneos quanto aos conceitos obtidos na avaliação Capes. Daí a importância da avaliação dos outros quesitos pela comissão da área que correspondem aos $65 \%$ restante da nota para atribuição do conceito dos programas.

\section{CONCLUSÃO}

Esta pesquisa teve dois objetivos: i. identificar como se agrupam os programas acadêmicos da área de Administração, Ciências Contábeis e Turismo utilizando somente a 


\section{A AVALIAÇÃO DE PROGRAMAS DA CAPES, OS CONCEITOS E O USO DE INDICADORES \\ BIBLIOGRÁFICOS \\ DOI: http://dx.doi.org/10.5007/1983-4535.2014v7n2p1}

produção científica veiculada em periódicos e ii. identificar como se associa o desempenho da produção científica veiculada em periódicos com o conceito Capes.

Para atingir o primeiro objetivo foi utilizada a análise de conglomerados nãohierárquica k-Means. $\mathrm{O}$ número de clusters predefinido foi de cinco para coincidir com o número categorias em que os programas recomendados pela Capes são classificados. Como input da análise realizada foram utilizados os indicadores de produção bibliográfica veiculada em periódicos contidos nas planilhas de indicadores da avaliação trienal da Capes. Os clusters criados possuíam tamanhos e composições distintas. Embora a área se subdivida em Administração, Ciências Contábeis e Turismo, apenas a categoria Turismo se agrupou integralmente dentro do mesmo cluster. Os clusters $1,2,3,4$ e 5 agruparam 8, 3, 3, 13 e 50 programas, respectivamente.

Os clusters 1, 3 e 4 agruparam a maioria dos programas com doutorado restando ao cluster 5 a predominância de programas que ofertam exclusivamente o mestrado. Os clusters 1, 3, 4 e 5 agruparam programas que receberam até três conceitos distintos pela Capes.

A relação da alocação dentro de um cluster e os conceitos Capes recebidos por um programa foram analisados por meio de uma Análise de Correspondência usando como inputs os clusters definidos com a análise de conglomerados. Tal análise permitiu identificar uma relação entre o conceito 4 e o cluster 2. Entretanto, não foi encontrada relação considerando os demais grupos formados.

Como a técnica utilizada é altamente sensível a inclusão ou exclusão de observações individuais sugere-se para pesquisas futuras: analisar programas com doutorado ou somente com mestrado em separado e relacionar com outras características dos programas, como idade, subárea, número de professores, e região geográfica ou estado.

\section{REFERÊNCIAS}

BEUREN, Ilse Maria; SOUZA, José Carlos de. Em busca de um delineamento de proposta para classificação dos periódicos internacionais de contabilidade para o Qualis CAPES. Revista Contabilidade \& Finanças, USP, São Paulo, v. 19, n. 46, p. 44-58, 2008.

CAPES - COORDENAÇÃO DE APERFEIÇOAMENTO DE PESSOAL DE NÍVEL SUPERIOR. Diretoria de Avaliação. Documento de Área 2013. Brasília: CAPES, 2013a. 56 p. Disponível em: $<$ http://www.capes.gov.br/images/stories/download/avaliacaotrienal/Docs_de_area/Administr a\%C3\%A7\%C3\%A3o_doc_area_e_comiss\%C3\%A3o_16out.pdf $>$. Acesso em: 28 jul. 2014. 


\section{A AVALIAÇÃO DE PROGRAMAS DA CAPES, OS CONCEITOS E O USO DE INDICADORES \\ BIBLIOGRÁFICOS \\ DOI: http://dx.doi.org/10.5007/1983-4535.2014v7n2p1}

CAPES - COORDENAÇÃO DE APERFEIÇOAMENTO DE PESSOAL DE NÍVEL SUPERIOR. Diretoria de Avaliação. Relatório de Avaliação 2010-2012 Trienal 2013. Brasília: CAPES, 2013b. 59 p. Disponível em: <

http://avaliacaotrienal2013.capes.gov.br/relatorios-deavaliacao/Administra\%C3\%A7\%C3\%A3o\%2C\%20Ci\%C3\%AAncias\%20Cont $\% \mathrm{C} 3 \% \mathrm{~A} 1$ beis $\% 20 \mathrm{e} \% 20$ Turismo.pdf?attredirects $=0 \& \mathrm{~d}=1>$. Acesso em: 28 jul. 2014.

CAPES - COORDENAÇÃO DE APERFEIÇOAMENTO DE PESSOAL DE NÍVEL SUPERIOR. Diretoria de Avaliação. Planilhas de Indicadores. Brasília: CAPES, 2014a. Disponível em: < http://avaliacaotrienal2013.capes.gov.br/resultados/planilhascomparativas/Administra $\% \mathrm{C} 3 \% \mathrm{~A} 7 \% \mathrm{C} 3 \% \mathrm{~A} 3 \mathrm{o} \% 2 \mathrm{C} \% 20 \mathrm{Ci} \% \mathrm{C} 3 \% \mathrm{AAncias} \% 20 \mathrm{Cont} \% \mathrm{C} 3 \% \mathrm{~A} 1$ beis $\% 20 \mathrm{e} \% 20$ Turismo.xls?attredirects $=0 \& \mathrm{~d}=1>$. Acesso em: 28 jul. 2014.

CAPES - COORDENAÇÃO DE APERFEIÇOAMENTO DE PESSOAL DE NÍVEL SUPERIOR. Sobre a Avaliação. Brasília: CAPES, 2014b. Disponível em: $<$ http://www.capes.gov.br/avaliacao/sobre-a-avaliacao>. Acesso em: 28 jul. 2014.

CAPES - COORDENAÇÃO DE APERFEIÇOAMENTO DE PESSOAL DE NÍVEL SUPERIOR. Qualis. Brasília: CAPES, 2014c. Disponível em:

$<$ http://www.capes.gov.br/avaliacao/instrumentos-de-apoio/classificacao-da-producaointelectual $>$. Acesso em: 28 jul. 2014.

COSTA, Abimael de Jesus Barros. Rankings dos programas de pós-graduação stricto sensu em Ciências Contábeis: análise da produção docente baseada em periódicos (2000 a 2009). Brasília, 2011. 126 p. Dissertação (Mestrado em Ciências Contábeis). Programa Multiinstitucional e Inter-Regional de Pós-Graduação em Ciências Contábeis da Universidade de Brasília - UnB, da, Universidade Federal da Paraíba - UFPB e da Universidade Federal do Rio Grande do Norte - UFRN.

FÁVERO, Luiz Paulo; BELFIORE, Patrícia; SILVA, Fabiane Lopes da; CHAN, Betty Lilian. Análise de dados. Modelagem multivariada para tomada de decisões. Rio de Janeiro: Campus, 2009.

IGARASHI, Deisy Cristina Corrêa et al. A qualidade do ensino sob o viés da avaliação de um programa de pós-graduação em contabilidade: proposta de estruturação de um modelo híbrido. Revista de Administração, v. 43, n. 2, p. 117-137, 2008.

LEITE FILHO, Geraldo Alemandro. Padrões de produtividade de autores em periódicos e congressos na área de contabilidade no Brasil: um estudo bibliométrico. RAC, v. 12, n. 2, p. 533-554, abr./jun. 2008.

LEITE FILHO, Geraldo Alemandro. Perfil da produção científica dos docentes e programas de pós-graduação em Ciências Contábeis no Brasil. Revista de Contabilidade e Controladoria, v. 2, n. 2, p. 1-13, mai./ago. 2009.

LINS, Marcos Pereira Estellita; ALMEIDA, Bernardo Faria de; BARTHOLO JUNIOR, Roberto. Avaliação de desempenho na pós-graduação utilizando a Análise Envoltória de 


\section{A AVALIAÇÃO DE PROGRAMAS DA CAPES, OS CONCEITOS E O USO DE INDICADORES \\ BIBLIOGRÁFICOS \\ DOI: http://dx.doi.org/10.5007/1983-4535.2014v7n2p1}

Dados: o caso da Engenharia de Produção. Revista Brasileira de Pós-Graduação, v. 1, n. 1, 2011.

MARTINS, Cibele. Desempenho dos programas de pós-graduação stricto sensu em administração, ciências contábeis e turismo no Brasil: um estudo dos programas no período de 2001 a 2009. São Paulo, 2013. 239 p. Tese (Doutorado em Administração). Programa de PósGraduação em Administração, Universidade Nove de Julho - UNINOVE.

MOREIRA, Carlos Otávio F.; HORTALE, Virginia Alonso; HARTZ, Zulmira de Araújo. Avaliação da pós-graduação: buscando consenso. Revista brasileira de pós-graduação, v. 1, n. $1,2004$.

NASCIMENTO, Sabrina do; BEUREN, Ilse Maria. Redes sociais na produção científica dos programas de pós-graduação de ciências contábeis do Brasil. Revista de Administração Contemporânea, v. 15, n. 1, p. 47-66, jan./fev. 2011.

ROCHA-E-SILVA, Mauricio. O Novo Qualis, que não tem nada a ver com a ciência do Brasil: carta aberta ao presidente da CAPES. Clinics, v. 64, p. 721-724, 2009.

SILVA, Harley Almeida Soares, REINA, Diane Rossi Maximiano; ENSSLIN, Sandra Rolim; REINA, Donizete. Programas de pós-graduação em contabilidade: análise da produção científica e redes de colaboração. Revista de Contabilidade e Organizações, v. 6, n. 14, p. 145-162, 2012.

SOARES, Sandro Vieira; CASA NOVA, Silvia Pereira de Castro; CASTRO JR, Francisco Henrique Figueiredo. O Qualis reflete o impacto dos artigos das Revistas Brasileiras de Contabilidade?. In: XVII SemeAd, 2014, São Paulo. Anais. São Paulo: USP, 2014.

SOARES, Sandro Vieira; PFITSCHER, Elisete Dahmer. Doutorado em Contabilidade no Brasil: há espaço para expansão da oferta de cursos? RBC: Revista Brasileira de Contabilidade, v. 195, p. 67-82, 2012.

SOARES, Sandro Vieira; RICHARTZ, Fernando; MURCIA, Fernando Dal-ri. Ranking da Pós-Graduação em Contabilidade no Brasil: Análise dos Programas de Mestrado com base na Produção Científica em Periódicos Acadêmicos no Triênio 2007-2009. Revista Universo Contábil, v. 9, n. 3, p. 55-74, jul./set., 2013.

SOARES, Sandro Vieira; WILL, Anderson Renan; MIRANDA, Maura Paula; PFITSCHER, Elisete Dahmer. Pós-Graduação em Ciências Contábeis no Brasil: Contexto e Processo de Seleção. Revista Ambiente Contábil, v. 3, p. 59-74, 2011.

SOUZA, Flávia Cruz, ROVER, Suliani, GALLON, Alessandra Vasconcelos, ENSSLIN, Sandra Rolim. Análise das IES da Área de Ciências Contábeis e de seus Pesquisadores por meio de sua Produção Científica. Contabilidade Vista \& Revista, v. 19, n. 3, p. 15-38, jul./set. 2008.

SPAGNOLO, Fernando; SOUZA, Valdinei Costa. O que mudar na avaliação da Capes?. Revista brasileira de pós-graduação, v. 1, n. 2, 2004. 
VIEIRA, Amanda Martins; ENSSLIN, Sandra Rolim; SILVA, Harley Almeida Soares da. Perfil da produção científica dos docentes dos departamentos de contabilidade de três universidades federais do sul do Brasil. Enfoque: Reflexão Contábil, v. 30, n. 3, p. 44-59, 2011. 